\title{
CdZnTe Material Uniformity and Coplanar-Grid Gamma-Ray Detector Performance
}

\author{
M. Amman, P. N. Luke, and J. S. Lee \\ Ernest Orlando Lawrence Berkeley National Laboratory \\ University of California, Berkeley, California 94720
}

\begin{abstract}
"Electron-only" detection techniques such as the coplanargrid method are effective in overcoming some of the charge transport problems of CdZnTe and, consequently, have led to large-volume gamma-ray detectors with good energy resolution while operating at room temperature. A requirement for the success of these techniques is uniformity in electron generation and transport. Once large inhomogeneities in these properties caused by grain boundaries and other large-scale crystal defects are eliminated through simple material screening techniques, small variations remain and limit the gamma-ray energy resolution. In this paper we present the results from our characterization and analysis of these nonuniformities in commercially available CdZnTe, and we establish the relationship between the nonuniformities and the ultimate coplanar-grid gamma-ray detector performance through experimental measurements.
\end{abstract}

\section{INTRODUCTION}

The potential for widespread application of $\mathrm{CdZnTe}$ materials to high-efficiency gamma-ray detection has been demonstrated through recent materials and detector developments [1-3]. The material, however, continues to suffer from poor hole transport leading to large-volume detectors with poor spectroscopic performance when conventional detection techniques are used. Consequently, detection techniques in which the detector signal is derived almost exclusively from electron collection have become the most viable approaches for successful application of the material to high-efficiency gamma-ray detection when good energy resolution is required. One such "electron-only" detection technique is the coplanar-grid charge-sensing technique [2]. This technique and its variations offer the advantages of near elimination of the hole collection problem, electron trapping compensation, uniform charge induction, and simplicity in the detection electronics.

Critical to the success of the coplanar-grid method and other electron-only techniques is uniformity in electron generation and transport in the CdZnTe material. Alphaparticle response measurements can be used to characterize these properties [4]. In one such method, an alpha-particle source is used to illuminate the cathode of a CdZnTe crystal that has been fabricated into a simple planar detector. Each alpha-particle interaction event in the detector generates a well-defined number of electron-hole pairs near the cathode. The collection of this charge produces an induced charge pulse on the detector electrodes that is almost entirely determined by the electron generation at the cathode and by the transport of the electrons through the entire detector length. The height of this pulse then characterizes the electron generation at the cathode and the electron transport along the drift path. Nonuniformities in these properties are reflected in the alpharesponse measurements as pulse-height variations. Consequently, a quick and accurate uniformity characterization of a crystal can be made by uniformly illuminating the full cathode area of the crystal and measuring the alpha-particle pulse-height spectrum. A spectrum that has a single sharp full-energy peak with little background indicates that the detector crystal is uniform in both the electron generation at the detector cathode and the electron transport throughout the crystal volume.

Further information concerning the inhomogeneities in the electron properties of a crystal can be obtained by finely collimating the alpha-particle source so that only a small volume of the crystal is probed. The alpha-particle spectral response of the crystal can then be measured as a function of the source location along the cathode. In this way a spatial map of the electron generation and transport can be made of the crystal thereby allowing the location of the inhomogeneities or the distribution of the variations to be determined in the two dimensions parallel to the cathode. Furthermore, analysis of the induced charge pulses at each source location can provide information concerning the depth location and nature of the electron transport inhomogeneities. Therefore, collimated source measurements combined with induced charge signal waveform measurements allow the inhomogeneities to be mapped in three dimensions and partially identified.

In an effort to better understand the primary factors that presently limit the gamma-ray detection performance of coplanar-grid detectors, we have characterized a large number of commercially available CdZnTe crystals. This characterization has consisted of alpha-particle response measurements of the type just described in addition to macroscopic defect identification through simple visual inspection and transmission infrared microscopy. The results of this characterization have then been compared to the coplanar-grid gamma-ray energy resolution of the crystals. In this paper we present the results of this study in which we have measured the uniformity of commercially available CdZnTe, determined the correlation between this uniformity and the ultimate coplanar-grid gamma-ray detection performance, and analyzed in detail some of the identified inhomogeneities. In the following section, we provide the details of our characterization procedure.

\section{Characterization PROCEDURE}

All of the results shown in this paper are from $1 \mathrm{~cm}^{3}$ CdZnTe crystals provided by eV Products [5]. Each of these crystals was processed through the same fabrication and 
characterization procedure which consists first of lapping the crystal surfaces with a fine-grit $\mathrm{SiC}$ powder in water slurry on a glass plate. The scattering of light from the lapped crystal surfaces is dependent on the crystallographic orientation thereby allowing the identification of grain boundaries through simple visual inspection of the surfaces. We crudely categorize a boundary as either a twin if it is straight or random if it is not. Following this, the crystal is mechanically polished with a water-based slurry of sub-micron alumina powder on a fabric pad in order to produce smooth surfaces. The crystal is then characterized using infrared transmission microscopy through which pipes, precipitates, and inclusions are identified. The fabrication of the crystal into a simple planar detector is continued by chemically etching the crystal in an approximately $2 \%$ bromine-methanol solution in order to remove the surface damage introduced by the mechanical processing. Immediately following the etch, full-area $\mathrm{Au}$ electrodes approximately $80-90 \mathrm{~nm}$ thick are deposited onto two opposing detector surfaces through thermal evaporation. The completed planar detector is then mounted into a vacuum chamber where one of the detector electrodes is illuminated with alpha particles from an ${ }^{241} \mathrm{Am}$ source. A thin windowless alpha-particle source is used, and the measurements are made under vacuum to ensure that the alpha particles entering the detector have a narrow energy distribution. A bias is applied across the detector to cause the collection of the electrons and holes generated by the alpha-particle interaction events within the detector. Finally, each of the charge pulses induced on the detector electrodes by the collection of the alpha-particle generated charge is measured with a charge-sensitive preamplifier and standard pulse-processing electronics chain. The use of an alpha-particle source provides the advantages of large signals, a well-defined energy deposition and interaction region (within $20 \mu \mathrm{m}$ of the entrance contact), and ease of collimation. These properties provide the means for highly detailed and accurate characterization of nonuniformities in charge generation and transport.

The measurement configuration just described is used to extract both the electron and hole mobilities and lifetimes of the CdZnTe crystal. For the electron properties, the cathode of the detector is illuminated with alpha particles, and pulses measured with a charge-sensitive preamplifier connected to the anode are acquired as a function of the detector bias. From each pulse a rise time $t_{r}$ and a pulse height $V_{p}$ are measured. This is done over a bias range of about $500 \mathrm{~V}$ to $2000 \mathrm{~V}$. Assuming a constant field in the detector, the electron mobility $\mu_{e}$ is determined from the rise time of each pulse using

$$
\mu_{e}=d^{2} / t_{r} V_{b}
$$

where $d$ is the detector thickness and $V_{b}$ is the magnitude of the bias applied across the detector. Typically the mobility is nearly constant over the bias range chosen. The electron mobility of the crystal is then taken as the average over the values extracted at the different biases. The electron lifetime is extracted assuming a single lifetime $\tau_{e}$ with no detrapping effects. The Hecht equation describes such a situation:

$$
V_{p}=\frac{Q_{o} \tau_{e}}{C_{f} t_{r}}\left(1-e^{-t_{r} / \tau_{e}}\right)
$$

where $Q_{o}$ is the amount of charge generated by an alphaparticle interaction event at the cathode and $C_{f}$ is the feedback capacitance of the charge-sensitive preamplifier. Equation (2) is fitted to the measured $V_{p}$ and $t_{r}$ data with $Q_{o} / C_{f}$ and $\tau_{e}$ as the fitting parameters thereby determining the electron lifetime of the crystal.

The hole mobility $\mu_{h}$ and lifetime $\tau_{h}$ of a CdZnTe crystal are determined using a similar measurement setup as that for the measurement of the electron properties. For the measurement of the hole properties, however, the anode of the detector is illuminated with alpha particles and the pulses induced on the cathode are measured. In this geometry the pulses are produced from the collection of the alpha-particle generated holes from the anode to the cathode. Since hole transport is poor in CdZnTe, the resultant small signal levels and long collection times complicate the extraction of the mobility and lifetime from this pulse data. For this reason, the mobility is determined from the initial slope of the pulse. The initial pulse slope is

$$
\frac{d V}{d t}(t=0)=\frac{Q_{o}}{C_{f} t_{r}}
$$

Substituting $t_{r}=d^{2} / \mu_{h} V_{b}$ into (3) and rearranging, we get the following for the hole mobility:

$$
\mu_{h}=\frac{d^{2}}{V_{b}} \frac{C_{f}}{Q_{o}} \frac{d V}{d t}(t=0) .
$$

Equation (4) allows us to directly determine the mobility from the initial slope of the induced charge signal pulse since the remaining parameters in the equation are known from the electron lifetime measurement.

The extraction of the hole lifetime is accomplished using a version of the Hecht equation which includes the effect of the exponential decay of our resistive-feedback charge-sensitive preamplifier. Using this equation, $t_{r}=d^{2} / \mu_{h} V_{b}$, the measured pulse height, and the previously extracted value of $\mu_{h}$, we obtain an equation which we solve numerically to determine $\tau_{h}$. We note that this method neglects detrapping effects which can be significant over the typical $20-50 \mu$ s rise times of the pulses. Consequently, the hole lifetime is overestimated in some cases. The extraction of $\mu_{h}$ and $\tau_{h}$ is made at a number of different biases, and the averages of these values are taken as the hole mobility and lifetime for the crystal.

Following these measurements of the average charge transport properties of the crystal, the overall electron generation and transport uniformity of the material is characterized. This is accomplished by uniformly illuminating the full cathode area of the crystal with an ${ }^{241} \mathrm{Am}$ alpha-particle source and measuring the resultant pulse-height spectrum at a typical detector bias of $1000 \mathrm{~V}$. The spectral line shape and any background that may be present provide a measure of uniformity.

One concern with this characterization method is that energy straggling from a dead layer at the entrance contact or a nonuniform dead layer could lead to a substantial broadening of the full-energy peak. If such broadening is large, then the full-energy peak width will be a measurement dominated by 


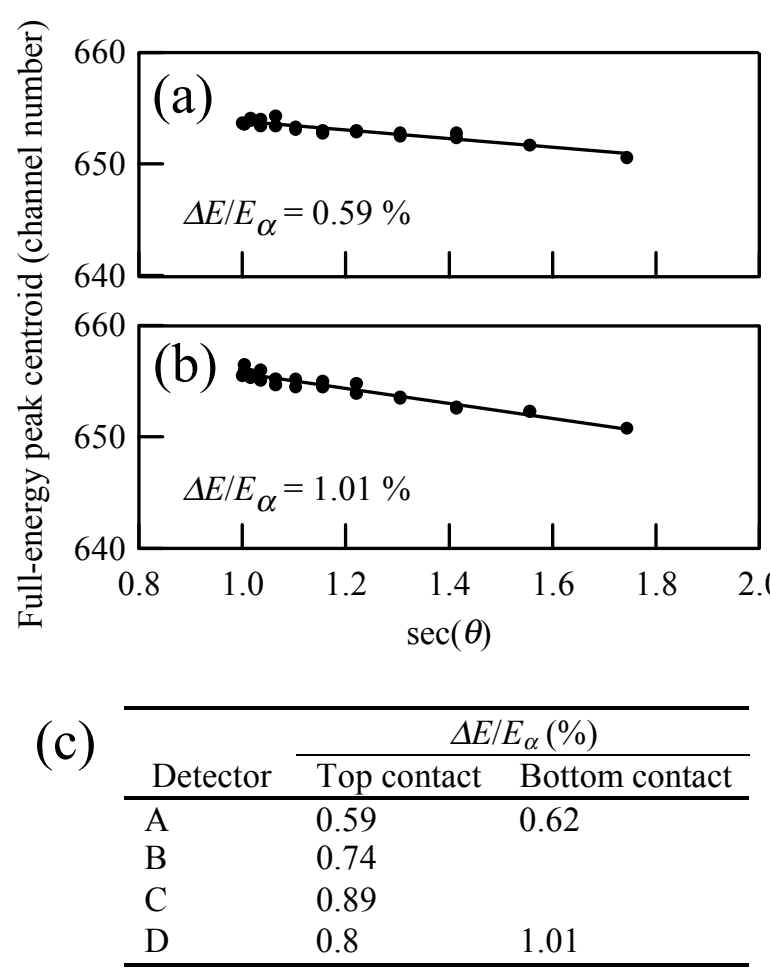

Figure 1. (a) and (b) Example contact thickness measurement data from two CdZnTe crystals with evaporated Au contacts. The fullenergy alpha-particle peak centroid measured with the planar geometry detectors is plotted as a function of the secant of the angle between the detector contact normal and the alpha-particle beam. An ${ }^{241} \mathrm{Am}$ alpha-particle source $(5.5 \mathrm{MeV})$ was used, and the measurements were made at a detector bias of $1000 \mathrm{~V}$. Ideally such a plot is linear, and the fraction of energy lost in the contact dead layer is the ratio of the slope magnitude to y-axis intercept. (c) Measured fraction of energy lost by $5.5 \mathrm{MeV}$ alpha particles in the contact dead layers of four CdZnTe crystals with evaporated Au contacts.

the dead layer and not the material nonuniformity. To find out if a contact dead layer is a problem, we determined the energy loss in the dead layer of the entrance contact by measuring the alpha-particle pulse-height spectrum from a collimated alphaparticle source as a function of the angle between the detector and the alpha-particle beam. If we assume that the rate of energy loss of the alpha particles in the Au contact layer and any CdZnTe dead layer is constant, then the pulse height is given by the following

$$
V_{p}=A\left(E_{\alpha}-\Delta E \sec (\theta)\right)
$$

where $A$ is a proportionality constant relating energy to pulse height, $E_{\alpha}$ is the alpha-particle energy, $\Delta E$ is the energy lost in the contact dead layer under normal incidence, and $\theta$ is the angle between the alpha-particle beam and the normal to the contact. Equation (5) is then fitted to the measured $V_{p}(\theta)$ data with $A E_{\alpha}$ and $A \Delta E$ as fitting parameters. The fraction of the energy lost in the entrance contact dead layer $\Delta E / E_{\alpha}$ is simply the ratio of these two fitted parameters. We have performed this measurement and analysis on four separate detectors. The data and results of the analysis are shown in Figure 1, where we have taken the average pulse height to be the centroid of the alpha-particle full-energy peak. The average fractional energy loss in the contact dead layer of about $0.8 \%$ is small and is comparable to the calculated loss of about $0.7 \%$ expected from the Au layer of the entrance contact [6]. Energy broadening due to this dead layer should be substantially smaller than this energy loss. We therefore expect the broadening due to the dead layer to be insignificant compared to the typical broadening we observe due to material nonuniformity.

Once the level of uniformity of a crystal is established through the full-area illumination alpha-particle response, we investigate the nature of the inhomogenieties in the electron generation and transport using alpha-particle scanning. For this measurement, the alpha-particle source is collimated to produce a beam diameter of about $1 \mathrm{~mm}$, and the source is mounted on a scanning stage so that the beam can be positioned anywhere along the cathode of the CdZnTe crystal. Both induced charge signals and pulse-height spectra are acquired as a function of the source location.

Following the above analysis of the crystal in the planar detector configuration, the crystal is reprocessed into a coplanar-grid detector and tested. The fabrication procedure is similar to that of the planar detector, except that the full-area anode is replaced with a coplanar-grid electrode structure that is defined by performing the Au evaporation through a shadow mask. The coplanar-grid pattern was designed for charge induction uniformity using three-dimensional electrostatic modeling and then later tested for uniformity through alphaparticle scanning measurements $[7,8]$. The completed coplanar-grid detector is placed inside a test chamber where it is illuminated with gamma rays from a ${ }^{137} \mathrm{Cs}$ source and operated as a differential-gain coplanar-grid detector [8]. The operating conditions of the detector are then optimized in order to produce the best possible $662 \mathrm{keV}$ gamma-ray peak resolution.

\section{RESULTS}

The value of using a simple planar geometry alpharesponse measurement for material characterization and screening is illustrated in Figure 2. Here the coplanar-grid detector gamma-ray energy resolution obtained from several CdZnTe crystals is plotted against the planar detector alphaparticle energy resolution obtained previously with these same crystals. There is a clear correlation between the two measurements. If the electron generation and transport of a crystal is highly nonuniform as is indicated by a poor planar detector alpha-particle energy resolution, then the crystal will lead to a coplanar-grid detector with poor gamma-ray energy resolution. The simple alpha-response measurement can therefore be used to screen material for coplanar-grid detector applications.

The correlation also implies that in many of the crystals the lack of uniformity in electron generation and transport is the limiting factor for the gamma-ray energy resolution obtained from the crystals. There are, however, other factors that can limit the gamma-ray performance of a crystal as a coplanargrid detector. For example, some crystals exhibit unusually high electronic noise as coplanar-grid detectors when full bias 


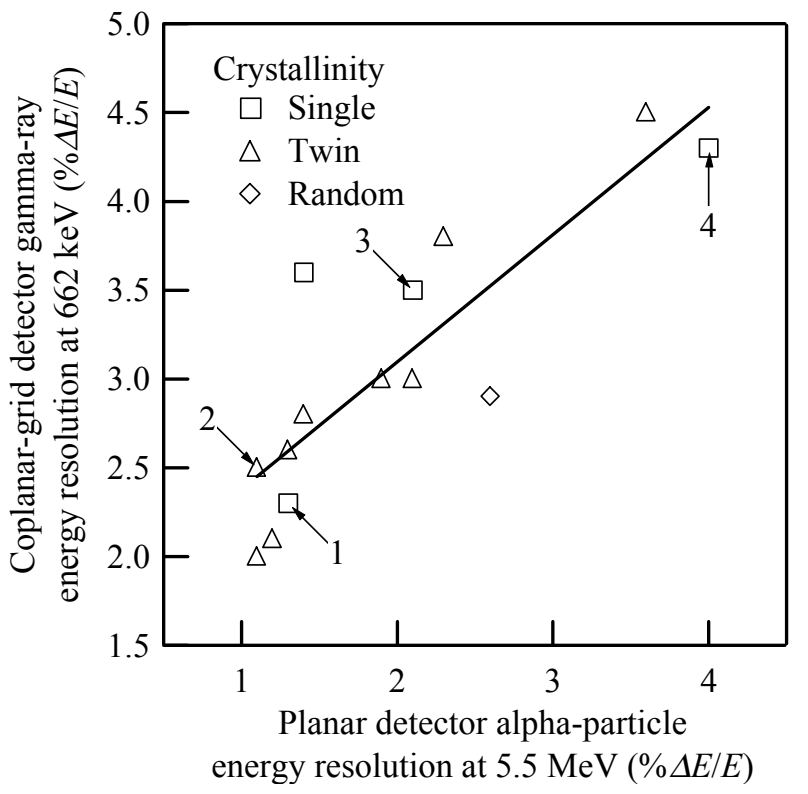

Figure 2. Measured coplanar-grid gamma-ray energy resolution at $662 \mathrm{keV}$ plotted against the planar detector alpha-particle energy resolution at $5.5 \mathrm{MeV}$ for $1 \mathrm{~cm}^{3} \mathrm{CdZnTe}$ crystals. The alpha-particle measurements were made at a bias of $1000 \mathrm{~V}$ whereas the gamma-ray measurements were made at biases that gave the best energy resolution.

is applied. The data from these crystals are not included in Figure 2 since this behavior prevents the proper optimization of the detectors. The electronic noise FWHM values for the crystals of Figure 2 operated as coplanar-grid detectors range from 8.7 to $14.7 \mathrm{keV}$ with an average value of $11.5 \mathrm{keV}$. This level of noise is still quite large in that the coplanar-grid gamma-ray energy resolution achieved with some of the more uniform crystals is significantly degraded by the noise. For example, three of the crystals of Figure 2 have resolutions of about $1.5 \%$ after the electronic noise has been subtracted. The electronic noise broadening is observed to be both fabrication process and material dependent.

We have also indicated in Figure 2 the presence of grain boundaries for each of the crystals. Only one of the crystals in the figure contained a randomly oriented grain boundary. The typically large electron trapping problems associated with such boundaries are well-established [9], and nearly all of the crystals that we tested with random boundaries produced results that were too poor to be included in Figure 2. Only a crystal in which these boundaries affected a small crosssection of the crystal produced a result that was good enough to fall within the range of the Figure 2 plot. The effect of these small boundaries is most likely a reduction in photo-peak efficiency. In contrast, the presence of large twin grain boundaries typically did not directly lead to a poor coplanargrid performance; though, as will be shown later, these boundaries may sometimes be associated with electron transport nonuniformities that degrade the coplanar-grid energy resolution. Fortunately, material can be screened for grain boundaries thereby eliminating the associated problems.

The crystals of Figure 2 have also been categorized based on pipe density and precipitate size and density (data not
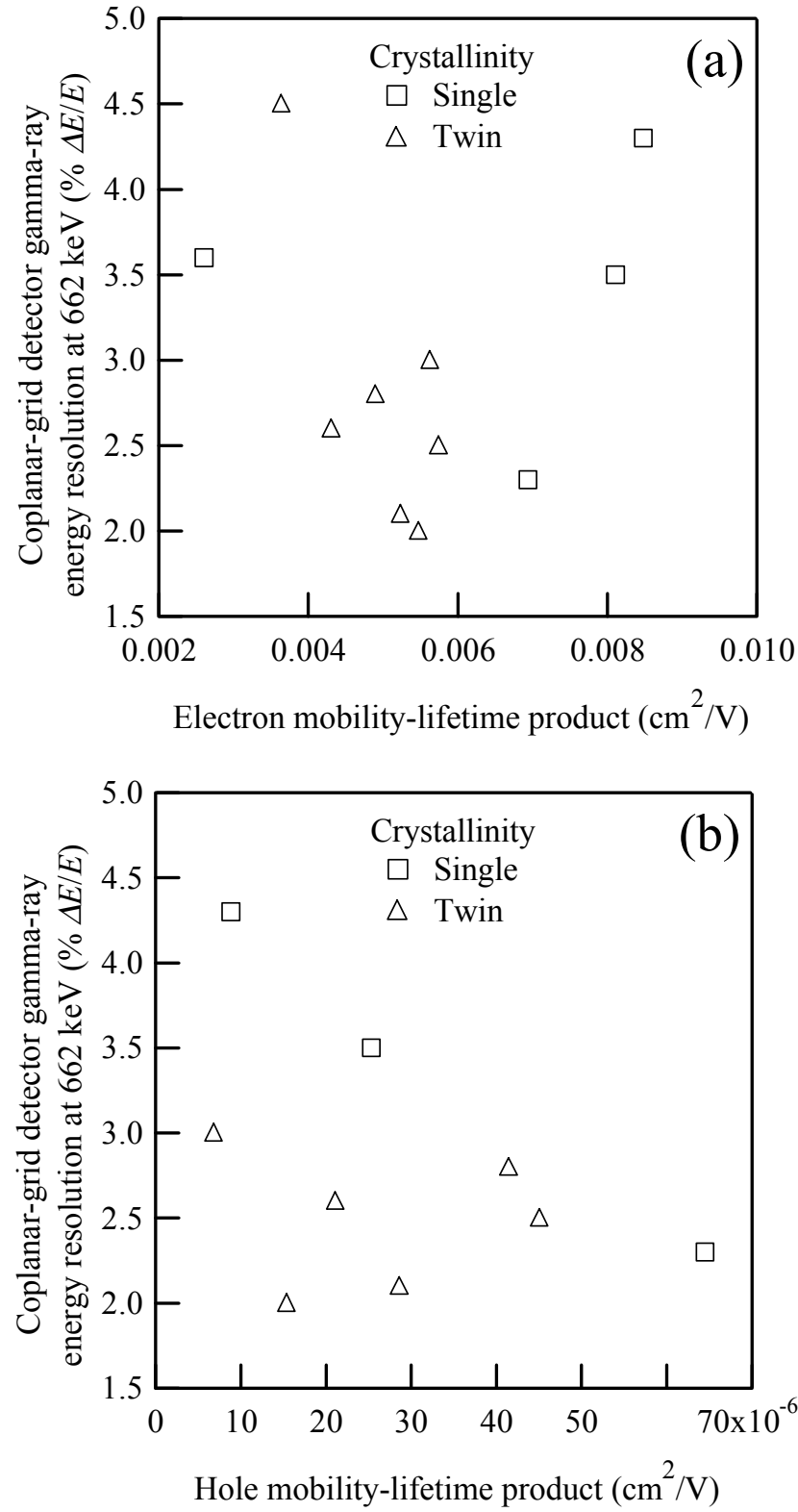

Figure 3. Measured coplanar-grid gamma-ray energy resolution at $662 \mathrm{keV}$ plotted against (a) electron mobility-lifetime product and (b) hole mobility-lifetime product for $1 \mathrm{~cm}^{3} \mathrm{CdZnTe}$ crystals.

shown). We do not see a good correlation between the pipe density and coplanar-grid gamma-ray detector performance. Based on this, it appears that the inhomogeneities in electron generation and transport, which are limiting the gamma-ray energy resolution, are not a direct result of the presence of pipes for these crystals. At this point a conclusion cannot be made concerning the impact of precipitate size and density, but there is some evidence that a high density of large precipitates causes problems with the coplanar-grid detector performance. More work needs to be done in this area of characterization.

Another possible correlation that we have investigated is that of the average carrier transport properties to coplanar-grid gamma-ray energy resolution. The results are shown in Figure 3 where the coplanar-grid gamma-ray energy resolution 

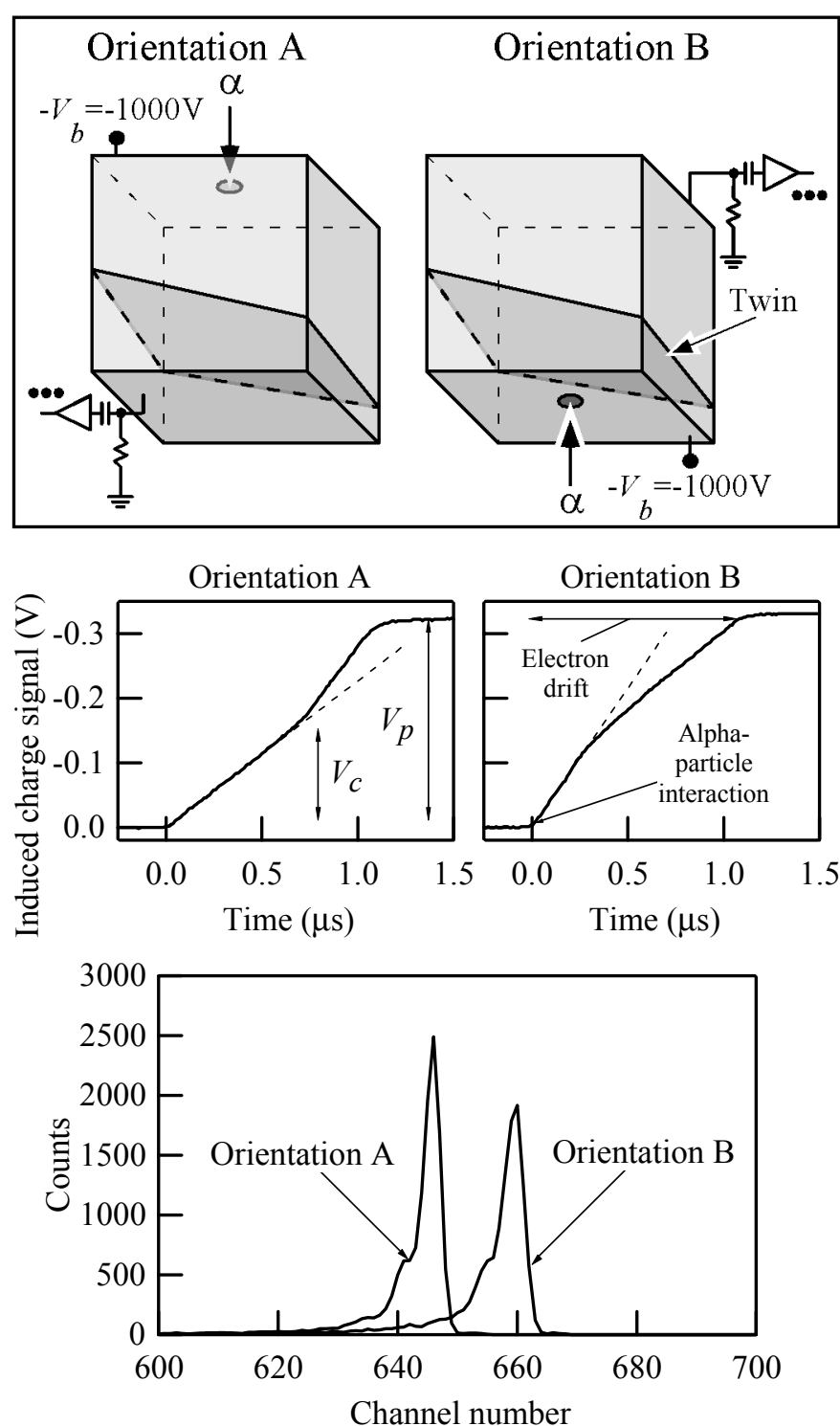

Figure 4. Top: Diagrams showing two configurations used to acquire data from crystal number 2 (performance indicated in Figure 2). The crystal was fabricated into a planar detector and illuminated with alpha particles from a collimated ${ }^{241} \mathrm{Am}$ source. The location of a twin in the crystal as identified through visual inspection of the lapped crystal surfaces is indicated. Middle: Typical induced charge signals measured from crystal number 2 using the two measurement configurations. Bottom: Superimposed pulse-height spectra acquired from crystal number 2 using the two measurement configurations.

is plotted against both the electron and the hole mobilitylifetime products. There is no clear correlation evident in either set of data. This is not surprising since, for the case of electron transport, the coplanar grid technique can accurately correct for the presence of uniform electron trapping. In principal, the magnitude of this uniform electron trapping could become a problem if it is large enough for the statistical fluctuations in the trapping to lead to a significant degradation in energy resolution. This does not appear to be the case. The uniformity rather than the average value of the electron transport (and generation) seems to be the factor limiting the coplanar-grid detector performance. No clear correlation with the average hole transport is also not surprising since the dependence of the detector signal on hole transport is largely eliminated through the coplanar-grid charge sensing technique. Some dependence on the hole transport could be possible, though, since if it is too poor, the trapped hole charge could lead to a polarization effect that would degrade resolution. Also, if the hole collection is too efficient, then the charge induction uniformity with depth is worsened when using the coplanar-grid technique, thereby degrading energy resolution [9]. Neither of these effects appears to be a limiting factor for the set of crystals analyzed.

To further understand the nature of the electron generation and transport inhomogeneities that degrade detector performance, we have used alpha-particle scanning combined with waveform analysis of the induced charge signals. An example set of results from the crystal labeled as 2 in Figure 2 is given in Figure 4. Measurements were made using two different detector orientations (Figure 4, top panel). In one, the alpha particles interact near the top contact of the detector, and the electrons generated by each interaction event drift to the bottom contact. In the other, the alpha particles interact near the bottom, and the generated electrons drift to the top. Despite the good $2.5 \%$ FWHM coplanar-grid gamma-ray energy resolution obtained with this crystal, a large electron transport inhomogeneity exists in this crystal as is evident from the induced charge signals of Figure 4. The signals from both orientations exhibit a large change in slope during the electron drift process. This slope is proportional to $\mu_{e} \mid \mathbf{E}$ (when trapping is neglected), where $|\mathbf{E}|$ is the magnitude of the electric field. These pulses are therefore consistent with the existence of two distinct regions of different $\mu_{e}|\mathbf{E}|$ within the detector. The larger $\mu_{e}|\mathbf{E}|$ region is the lower portion of the detector. For such a situation, we would expect that a larger pulse height would result for orientation $\mathrm{B}$ in which the electrons drift through the high $\mu_{e}|\mathbf{E}|$ region first. This is because less charge loss due to trapping occurs in the high $\mu_{e}|\mathbf{E}|$ region. Therefore more of the initially generated electrons will drift a greater distance through the crystal in orientation $\mathrm{B}$ than in $\mathrm{A}$, thereby producing a greater pulse height. This effect is shown in the pulse-height spectral measurements of Figure 4. The rather large difference in $\mu_{e}|\mathbf{E}|$ between the two regions of about $50 \%$ amounts to only a $2.1 \%$ difference in full-energy peak position. This measured pulse-height difference is consistent with that calculated from a simple Hecht-equation type model from which a $2.6 \%$ pulse-height difference is obtained.

Crystal number 2 contains a twin boundary, which is schematically indicated in the upper diagrams of Figure 4. To determine if the boundary between the two regions with different $\mu_{e}|\mathbf{E}|$ coincides with the twin boundary, the location of the $\mu_{e}|\mathbf{E}|$ change can be estimated using

$$
d_{c}=\frac{V_{c}}{V_{p}} d
$$

where $d_{c}$ is the depth from the entrance contact at which the $\mu_{e}|\mathbf{E}|$ change occurs and $V_{c}$ is the signal amplitude when the change occurs. By performing this waveform analysis for a 


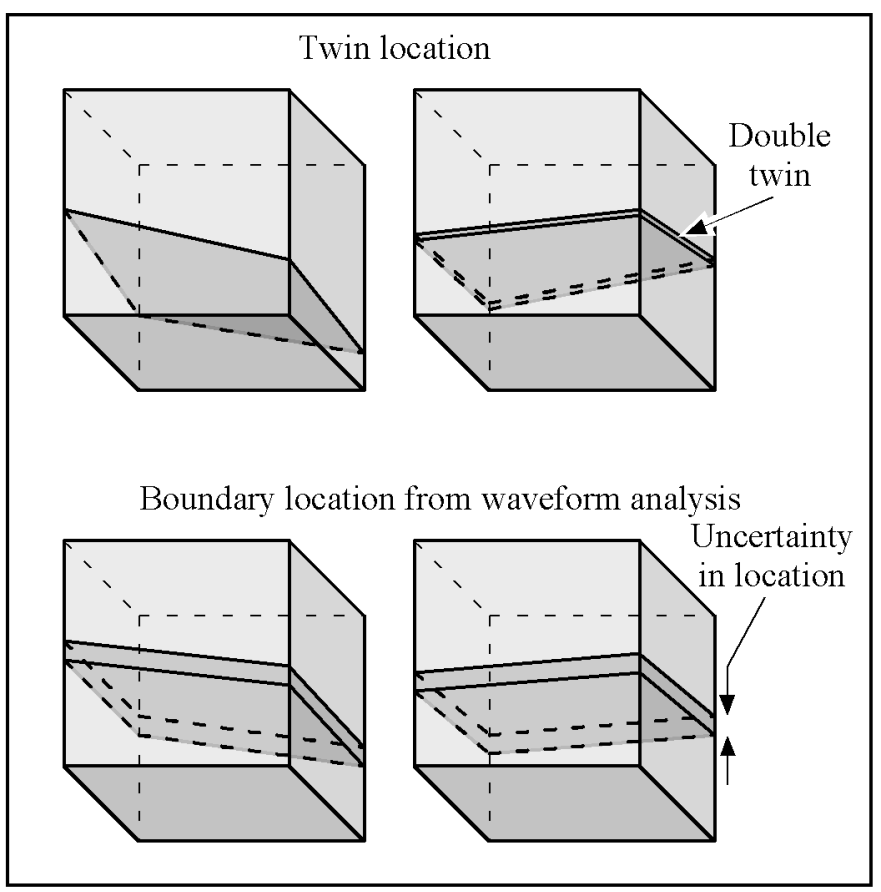

Figure 5. Top: Diagrams indicating the locations of twin planes for two CdZnTe crystals. The diagram on the left is from crystal number 2. Bottom: Diagrams indicating the locations of the mobility-field change within the two crystals whose twin boundaries are diagramed in the top portion of this figure. The mobility-field change boundaries have been mapped using alpha-particle scanning combined with waveform analysis. The uncertainty in boundary location is a result of a difference in the extracted boundary location between the two measurement orientations depicted in Figure 4.

number of locations of the alpha-particle source along the detector cathode, the boundary can be located in three dimensions. We have performed this mapping analysis on crystal number 2 and another crystal that also contains a twin boundary and exhibits the two-region behavior. The results are shown in Figure 5. There does appear to be a correlation between the twin location and the $\mu_{e}|\mathbf{E}|$ change, however the two boundaries do not always coincide as the crystal number 2 diagrams indicate. Furthermore, the two region $\mu_{e}|\mathbf{E}|$ behavior has also been observed in an apparently single crystal piece of material, though the magnitude of the $\mu_{e}|\mathbf{E}|$ change between the regions was substantially less than that of the two crystals with twin boundaries shown here.

The impact that the $\mu_{e}|\mathbf{E}|$ change has on coplanar-grid gamma-ray energy resolution can be estimated through simple detector modeling of the type described in previous studies $[8,10]$. Using the $\mu_{e}|\mathbf{E}|$ numbers extracted from the data of Figure 4 and including the measured electronic noise of the coplanar-grid detector of $12.9 \mathrm{keV}$ FWHM, a detector resolution of $16.0 \mathrm{keV}$ FWHM was obtained from the model. This is nearly the same as the actual $16.3 \mathrm{keV}$ FWHM value measured with the detector. For the case of uniform $\mu_{e}|\mathbf{E}|$, the model predicts a resolution of $12.9 \mathrm{keV}$, in other words, one that is dominated entirely by electronic noise. Therefore it is reasonable to conclude that much of the resolution broadening in crystal number 2 that is in addition to that of the electronic

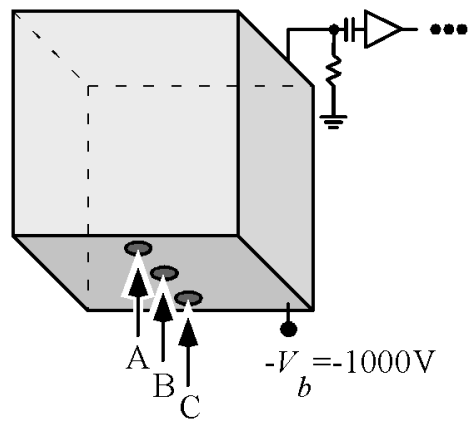

$\alpha$ source locations

Figure 6. Measurement configuration used to acquire the data shown in Figures 7 and 8. Separate measurements were made at the three alpha-particle source locations indicated. The source locations are separated by $2 \mathrm{~mm}$.

noise is a direct result of the $\mu_{e}|\mathbf{E}|$ nonuniformity. At this point in our study, the physical origin of this nonuniformity is unknown.

Variations in $\mu_{e}|\mathbf{E}|$ of the type just analyzed can impact coplanar-grid energy resolution, however they do not seem to be the primary cause leading to the results of Figure 2. The resultant degradation is too small, and for nearly all of the crystals of Figure 2, variations in $\mu_{e}|\mathbf{E}|$ are much less than that observed in crystal number 2 .

Further insight into the nature of the nonuniformities leading to gamma-ray energy resolution degradation is provided by the alpha-particle scanning measurements (configuration shown in Figure 6) given in Figures 7 and 8. In these figures the results from the single crystals labeled 1 and 3 in Figure 2 are shown. For each crystal the alpha-particle response has been measured at three different source locations along the detector cathode. As indicated in Figure 2, crystal number 1 achieved a good coplanar-grid gamma-ray resolution of $2.3 \%$ FWHM at $662 \mathrm{keV}$, yet the alpha-particle spectra of Figure 7(a) clearly indicate the presence of some nonuniformity. Each individual spectrum has a sharp peak with better than $0.6 \%$ FWHM energy resolution indicating good uniformity over the length-scale of the collimator size (about $1 \mathrm{~mm}$ ), however the peaks are shifted from each other. The crystal exhibits a long length scale nonuniformity. Typical induced charge signals aquired at each source location are superimposed and plotted in Figure 7(b). The pulses do not exhibit any gross slope changes of the type observed with crystal number 2 (Figure 4). There are small wiggles in the nearly linear rise of the pulses that distinguish them from each other. However, if these wiggles are simply a result of small $\mu_{e}|\mathbf{E}|$ variations, they could not possibly explain the variation in pulse heights between the different locations. As the crystal number 2 example has shown, a large variation in $\mu_{e}|\mathbf{E}|$ is required in order to produce a peak shift of the order of that shown in Figure 7(a). It is more likely that the peak shifts result from a difference in the amount of charge generated by the alpha particles at the different source locations or by a 

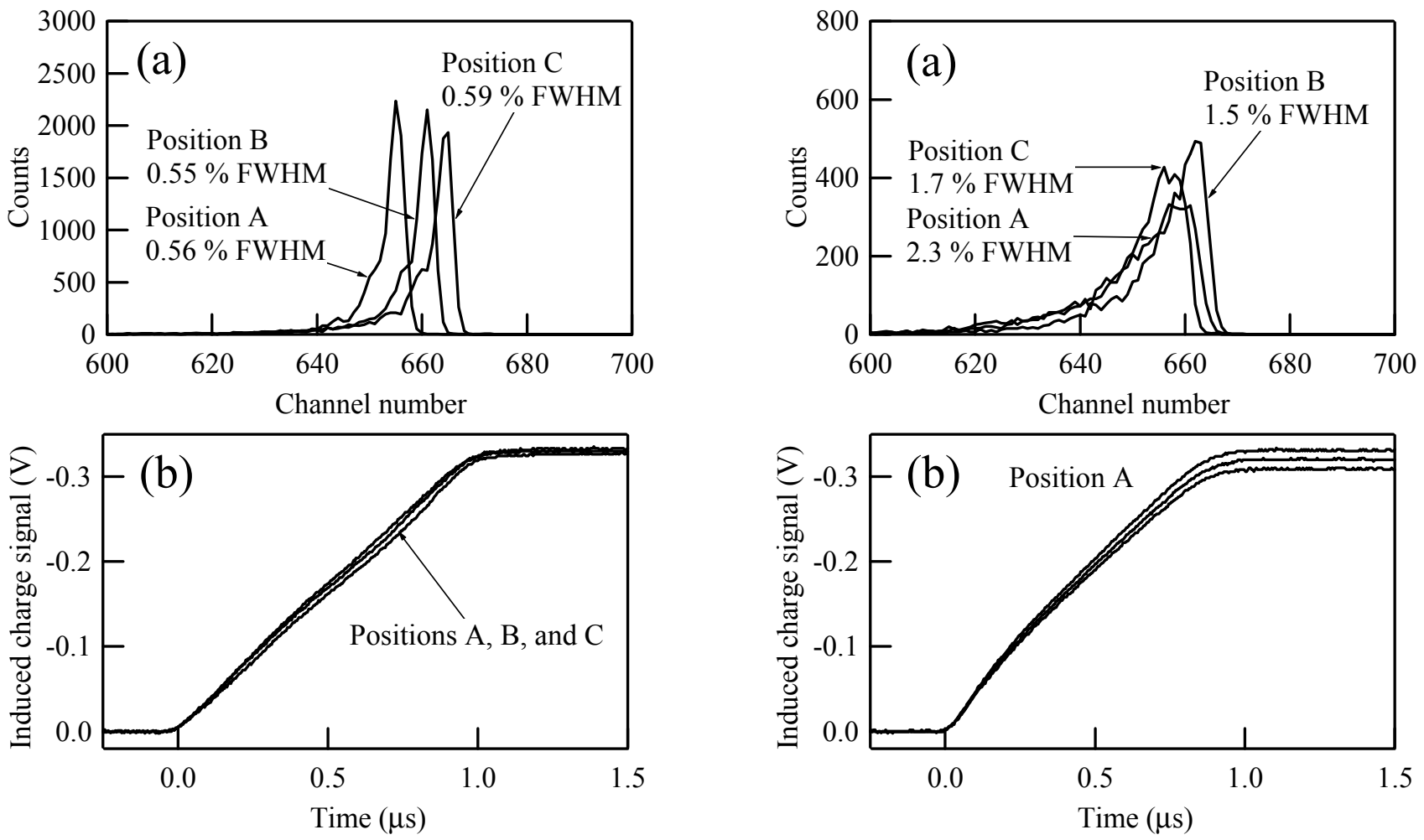

Figure 7. (a) Superimposed alpha-particle pulse-height spectra obtained at the source locations indicated in Figure 6 from the crystal labeled number 1 in Figure 2. The crystal was fabricated into a planar detector and illuminated with alpha particles from a collimated ${ }^{241} \mathrm{Am}$ source. (b) Superimposed induced charge signals acquired using crystal number 1 for the three alpha-particle source locations.

difference in the level of charge trapping between the different locations.

A crystal with more substantial nonuniformities is crystal number 3 whose alpha-particle scanning results are shown in Figure 8 . In contrast to crystal number 1 , each alpha-particle spectrum acquired with this crystal has a broad peak with a width of at least $1.5 \%$ FWHM. The crystal therefore has significant inhomogeneities over the length scale of less than $1 \mathrm{~mm}$. These small length scale nonuniformities are most likely the cause of the mediocre $3.5 \%$ FWHM coplanar-grid gamma-ray energy resolution obtained with this crystal. This idea is also substantiated by crystal number 4 (identified in Figure 2) which has even more severe small length scale nonuniformities as evidenced by typical scanning alphaparticle peak resolutions greater than $2.5 \%$ FWHM (data not shown), and as expected, the coplanar-grid gamma-ray detector performance is poor with only a $4.3 \%$ FWHM resolution being achieved.

A typical set of induced charge pulses obtained from crystal number 3 is shown in Figure 8(b). All of the pulses were acquired with the source at one location. The pulses all have a similar shape again indicating that $\mu_{e}|\mathbf{E}|$ variations are probably not the cause for the observed pulse-height differences. The investigation into the origin of these small length scale nonuniformities is on going.

Figure 8. (a) Superimposed alpha-particle pulse-height spectra obtained at the source locations indicated in Figure 6 from the crystal labeled number 3 in Figure 2. The crystal was fabricated into a planar detector and illuminated with alpha particles from a collimated ${ }^{241} \mathrm{Am}$ source. (b) Typical set of induced charge signals acquired using crystal number 3 with the source located at position A.

\section{SUMMARY}

In this paper we have more fully established the relationship between the uniformity in electron properties of $\mathrm{CdZnTe}$ material and the coplanar-grid gamma-ray detector performance of the material. The electron generation and transport uniformity of a number of CdZnTe crystals was characterized through a simple alpha-particle response measurement. These results were then compared to the coplanar-grid gamma-ray performance of these same crystals. A good correlation between the two measurements was found thereby indicating that a primary problem limiting the performance of coplanar-grid detectors is uniformity in electron generation and transport. A detailed investigation into the origin of these material inhomogeneities was pursued through the use of alpha-particle scanning combined with pulse-height and waveform analyses. One type of nonuniformity, that of a change in the product of electron mobility and detector field, was identified and its location mapped in three dimensions. A sufficiently large extent of such variations was shown to degrade coplanar-grid detector performance, however these nonuniformities do not appear to be the dominant material inhomogeneity for the majority of the crystals studied. Nonuniformities apparently not explainable through simple mobility-field changes were also characterized through the scanning techniques. The more severe of these have substantial variations over small length scales (less than 
Presented at the 1999 IEEE Nuclear Science Symposium

$1 \mathrm{~mm}$ ) and were observed in single crystals that produced poor coplanar-grid detector performances. The next step in our study is to correlate these alpha-particle response measurements with the results from other materials characterization techniques in order to discover the fundamental physical origin of the nonuniformities that presently limit the performance of coplanar-grid detectors.

\section{ACKNOWLEDGMENTS}

This work is supported by the U.S. Department of Energy, Office of Nonproliferation \& National Security, NN20.

\section{REFERENCES}

[1] Semiconductors for Room Temperature Nuclear Detector Applications, Semiconductors and Semimetals, vol. 43, edited by T. E. Schlesinger and R. B. James, Academic Press, New York, 1995.

[2] P. N. Luke, "Unipolar charge sensing with coplanar electrodes - application to semiconductor detectors," IEEE Trans. Nucl. Sci., vol. 42, pp. 207-213, 1995.

[3] Z. He, G. F. Knoll, D. K. Wehe et al., "1-d position sensitive single carrier semiconductor detectors," Nucl. Instrum. Methods Phys. Res., vol. A380, pp. 228-231, 1996.
[4] M. Amman, J. S. Lee, and P. N. Luke, "Electron transport uniformity characterization of $\mathrm{CdZnTe}$ using alpha particles," SPIE, vol. 3446, pp. 179-190, 1998.

[5] eV Products, a division of II-VI, Inc., Saxonburg, PA 16056.

[6] SRIM program described in The Stopping and Range of Ions in Solids, by J. F. Ziegler, J. P. Biersack, and U. Littmark, Pergamon Press, New York, 1985.

[7] P. N. Luke, M. Amman, T. H. Prettyman, P. A. Russo, and D. A. Close, "Electrode design for coplanar-grid detectors," IEEE Trans. Nucl. Sci., vol. 44, pp. 713-720, 1997.

[8] M. Amman and P. N. Luke, "Optimization criteria for coplanar-grid detectors," IEEE Trans. Nucl. Sci., vol. 46, pp. 205-212, 1999.

[9] P. N. Luke and E. E. Eissler, "Performance of CdZnTe coplanar-grid gamma-ray detectors," IEEE Trans. Nucl. Sci., vol. 43, pp. 1481-1486, 1996.

[10] M. Amman and P. N. Luke, "Coplanar-grid detector with single-electrode readout," SPIE, vol. 3115, pp. 205-213, 1997. 